\title{
Dynamique des peuplements des Parcelles d'Essais Sylvicoles (PES) de la forêt classée de la Sota au Nord-Est du Bénin
}

\author{
Gaston Sèhounkpindo AKOUEHOU ${ }^{1 *}$, Romaric Serge LOKOSSOU ${ }^{1}$, \\ Ingrid Sènan $\mathrm{LEEBA}^{1}$, Idrissou Chabi $\mathrm{BIO}^{1}$ et Léon Zoundo YAOU ${ }^{2}$ \\ ${ }^{I}$ Centre d'Études, de Recherche et de Formation Forestières (CERF), Bénin. \\ ${ }^{2}$ Direction Générale des Forêts et Ressources Naturelles (DGFRN), Bénin. \\ *Auteur correspondant, E-mail: akouehougas@gmail.com; Tel:(+314) 747-0240; Fax: (+314) 454-8294.
}

\section{RESUME}

L'étude de la dynamique des populations est essentielle pour le développement de scénarios de gestion rationnelle des écosystèmes forestiers. Cette étude réalisée sur des parcelles expérimentales forestières permanentes (PFTP) à Bensékou visait à évaluer la dynamique de la population de la forêt classée de Sota, sous la menace de la pression anthropique, par l'analyse du développement des peuplements sur PFTP au Nord-Est du Bénin. Ce travail a permis d'obtenir un inventaire floristique (identification des espèces, mesure de DBH) sur ce PFTP en lisière de forêt. Le traitement To (sans traitement) et T2 T1 (simple Coppice) ont été appliqués. De plus, des données historiques sur l'évolution de PFTP depuis 1996 ont été considérées. Un total de 09 espèces regroupées en 05 familles ont été identifiées. Les Fabaceae forment le fonds floristique. La formation est une savane arbustive, dominée par Detarium microcarpum, Terminalia avicennioides, Vitellaria paradoxa, Combretum racemosum et en minorité par Prosopis africana, Vitex doniana et Pterocarpus erinaceus. Les espèces à croissance relativement rapide sont Detarium microcarpum, Vitellaria paradoxa, Lannea microcarpa, Pterocarpus erinaceus et Terminalia avicennioides. Dans les sous-traitements T1 et T2 regroupés dans les parcelles T3, une augmentation de la densité moyenne d'environ 772 pieds / ha entre 1996 et 2014 a été constatée, ce qui s'explique par la forte capacité de régénération. La parcelle sous traitement A a également une augmentation de 428 pieds / ha dans la même période. En ce qui concerne la surface terrière, on constate une augmentation d'environ $20 \mathrm{~m}^{2} /$ ha entre 1996 et 2001 , pour tomber brutalement à $2,68 \mathrm{~m}^{2} /$ ha de 2001 à 2014. Les parcelles traitées ont donc une dynamique évolutive plus rapide; mais la pression anthropique exercée par la population riveraine ne permet pas aux arbres d'avoir un grand diamètre avant d'être abattus. Il est alors important de mettre en œuvre une sensibilisation de la population locale sur les mérites de la gestion rationnelle de cette formation végétale sur leur vie et sur l'environnement.

(C) 2017 International Formulae Group. All rights reserved.

Mots clés: Dynamique des populations, parcelles permanentes d'essais forestiers (PFTP), régénération naturelle, forêt classée de Sota, Nord-est du Bénin.

\section{Dynamics stands of Woodland Testing Plots (WTP) of the Sota Forest in North-East Benin}

\begin{abstract}
Population dynamics study is an essential for the development of rational management scenarios of forest ecosystems. This, This study carried out on permanent Forestry trials plots (PFTP) at Bensékou aimed at
\end{abstract}


assessing the population dynamics of the Sota classified forest, under the threat of anthropogenic pressure, by the analysis of stand development on PFTP in the North East of Benin. This work achieved a floristic inventory (species identification, DBH measuring) on this PFTP on the edge of the forest. The To treatment (without treatment) and T2 T1 (single Coppice) were applied. Moreover, historical data about the evolution of PFTP since 1996 were considered. A total of 09 species grouped into 05 families were identified. The fabaceae form the floristic fund. The formation is a shrub savanna, dominated by Detarium microcarpum, Terminalia avicennioides, Vitellaria paradoxa, Combretum racemosum and in minority by Prosopis africana and Vitex doniana, Pterocarpus erinaceus. The relatively fast-growing species are Detarium microcarpum, Vitellaria paradoxa, Lannea microcarpa, Pterocarpus erinaceus, and Terminalia avicennioides. In under treatments T1 and T2 grouped in plots T3, an increase of the average density for approximately 772 feets/ha between 1996 and 2014 was noted, which is explained by the strong capacity for regeneration. The plot under To treatment also have an increasing of $428 \mathrm{feet} / \mathrm{ha}$ in the same period. Regarding the basal area, we notice an increase of about $20 \mathrm{~m}^{2}$ /ha between 1996 and 2001, then abruptly drops to $2.68 \mathrm{~m}^{2} /$ ha from 2001 to 2014 . Therefore, the plots which have been treated had a faster evolutionary dynamics; but anthropogenic pressure exerted by the riparian population does not allow trees to have a large diameter before being cut down. It is then important to implement awareness raising of the local population on the merits of rational management of this plant formation on their lives and on the environment.

(C) 2017 International Formulae Group. All rights reserved.

Keywords: Population dynamics, permanent Forestry trials plots (PFTP), natural regeneration, classified forest of Sota, North East of Benin.

\section{INTRODUCTION}

De nombreux inventaires forestiers traditionnels ont souvent mis l'accent sur le potentiel d'exploitation $\mathrm{du}$ bois et ne fournissent donc que peu d'informations ou aucune sur les arbres de plus petite taille (Tiokeng et al., 2015). L'inventaire réalisé par le projet Aménagement Participatif dans la forêt de la Sota en 1996 avait permis d'apprécier les caractéristiques dendrométriques des différentes strates par espèce . Il fallait mettre au point une méthode de conversion des peuplements présentant de nombreuses espèces en des peuplements à espèces de valeurs en bois. Cette conversion a nécessité de connaître le potentiel de productivité et les méthodes simples de coupe et de régénération. Car selon Hitimana et al. (2004) cité par Yehouenou et al. (2012), les contraintes à la gestion durable des forêts en Afrique Subsaharienne sont entre autres la connaissance limitée de l'état des forêts et de leurs conditions et fonctions en termes de structure, composition, régénération et l'évaluation quantitative des bénéfices tangibles et intangibles qu'on peut en tirer.

Ainsi, dans le cas du taillis, il s'agissait de connaître les paramètres qui vont permettre de fixer la rotation et le volume attendu et de proposer éventuellement des méthodes spécifiques et simples de coupe et de régénération. Il était alors question de connaître les forêts, leur production (surtout en bois) et la manière de conduire les interventions sylvicoles (coupe et régénération naturelle).

Ainsi, après l'inventaire complet et la coupe rase en 1996, les mensurations des variables retenues se sont poursuivies en 1997 et 1998. Des relevés ont été également effectués en 2004.

En effet, une connaissance minimum de la dynamique des peuplements, perturbés par des interventions humaines, constitue un élément indispensable à l'élaboration de scénarios de gestion raisonnée des écosystèmes de forêt dense tropicale (Favrichon et al., 1998). Le suivi de la régénération naturelle est fondamental 
(Alexandre, 1989); Car selon Lakany (2004) cité par Akouehou et al. (2013), bien que l'importance du rôle des forêts dans la lutte contre la pauvreté et la protection de l'environnement soit de plus en plus reconnue, la déforestation et la dégradation des forêts se poursuivent dans des proportions alarmantes. La maitrise de la dynamique de population permettra de suivre l'ensemble des changements qui se produisent au cours de la vie d'une population (Frontier et al., 1991; Ngueguim, 2009). Mais les études réalisées par Zobel et al. (1989) sur différentes espèces montrent que la densité de peuplement joue un rôle sur la croissance de l'arbre, en agissant sur la compétition entre les arbres d'un même peuplement.

Partant, des informations recueillies durant cette étude, il apparaît que trois volets devraient faire l'objet d'études approfondies, après une synthèse bibliographique sur la dynamique de cette végétation naturelle. Suivi sur le moyen et long terme, de la dynamique de la végétation. Après un état des lieux, les relevés doivent se poursuivre sur une période de l'ordre de la décennie au moins. Des sites caractéristiques des différents types végétaux. Un inventaire floristique et structural quantitatif sur les espèces ligneuses et herbacées sera répété tous les deux à cinq ans

L'objectif principal de cette étude est de faire la table de productivité des espèces autochtones en zone de savane. Il s'agira spécifiquement i) d'évaluer les paramètres dendrométriques ainsi que la richesse floristique du peuplement ; ii) d'évaluer la dynamique des peuplements de la forêt classée de la Sota à travers les Parcelles d'Essais Sylvicoles et iii) d'analyser la croissance et la production des peuplements au regard des différents traitements sylvicoles sous l'effet de la pression anthropique.

\section{MATÉRIEL ET MÉTHODES \\ Description de la Forêt Classée de la Sota}

La forêt classée de la Sota est située au nord-est du Bénin et s'étend entre les parallèles $10^{\circ} 58$ et $11^{\circ} 11$ de latitude nord d'une part et les méridiens $3^{\circ} 03$ et $3^{\circ} 25$ de longitude, d'autre part. Conformément à l'Arrêté $\mathrm{N}^{0} 1862 / \mathrm{SE}$ du 16 mai 1947, la forêt domaniale de la couvre une superficie d'environ 53000 ha (Figure 1).

\section{Méthodes de collecte et de traitements Méthode de collecte}

La méthodologie utilisée a consisté à faire un inventaire forestier basé sur les mensurations (identification et mesure du $\mathrm{DBH})$ après avoir identifié et délimité les unités de relevés. En effet, les placettes ont été identifiées à l'aide du dispositif expérimental schématisé et imprimé sur feuille. A ce niveau, le dispositif qui existait a fait l'objet de modification car seulement deux traitements (To : témoin ou pas de traitement, T1 et T2 : (T3) Taillis simple) ont été considérés, ce qui a fait diminué le nombre de placette au total 18 à 9 disposées et 3 blocs. La délimitation a consisté à fusionner les 18 anciennes sous placettes de dimension $25 \mathrm{~m} \mathrm{X}$ $50 \mathrm{~m}$ en 9 placettes de dimension $56 \mathrm{~m} \mathrm{X} 50$ $\mathrm{m}$. Des coupes systématiques des essences ont été effectuées pour dégager les allés de $10 \mathrm{~m}$ de large qui séparent les différents blocs et placettes (Figure 2).

\section{Dispositif expérimental}

Les collectes ont été réalisées dans un dispositif dont la taille est $25200 \mathrm{~m}^{2}(150 \mathrm{~m} \mathrm{x}$ $168 \mathrm{~m})$ pour l'essai. Chaque unité 
expérimentale est entourée d'un couloir de 10 mètres et l'ensemble est entouré d'un couloir de 30 mètres non cumulatif. De façon physique, nous avons en face un dispositif de dimensions 248 x 230 mètres. Pour les mensurations et l'aménagement, nous avons utilisé des rubans pi, de ruban couturier, d'un penta décamètre, d'une boussole, de 2 GPS, des fiches d'inventaire, d'écritoires, de gommes, de deux machettes, d'un moyen de transport. Les informations sont traitées et analysées pour contribuer à la reconstitution de l'histoire de la croissance des sujets des parcelles d'essai, ce qui permet de connaître la dynamique du peuplement (Figure 2).

\section{Méthode d'analyse}

La description complète de la diversité floristique est fournie par un tableau de présence-absence ou du nombre d'individus par espèce et par unité d'échantillonnage (Fandohan, 2007). Ils présentent un intérêt à la fois pour comparer différents sites entre eux (ou même les différentes parcelles du dispositif avant traitement), mais également pour évaluer l'impact à court, moyen et long terme des perturbations humaines sur le dispositif (Favrichon et al., 1998).

$\checkmark \quad$ Description complète de la diversité floristique.

La description est fournie par un tableau de présence-absence ou du nombre d'individus par espèce et par unité d'échantillonnage (21). Ils présentent un intérêt à la fois pour comparer différents sites entre eux (ou même les différentes parcelles du dispositif avant traitement), mais également pour évaluer l'impact à court, moyen et long terme des perturbations humaines sur le dispositif (1).

Densité du peuplement - Selon le programme national des données sur les forêts du Canada (http://nfdp.ccfm.org/) la densité de peuplement est une mesure quantitative du couvert forestier sur une superficie donnée.
Elle s'exprime en fonction de la biomasse, de la fermeture du couvert, du nombre d'arbres, de la surface terrière ou du volume par hectare. Dans le cas présent, nous le définissons comme le nombre de pieds d'arbre ramené à l'hectare $(\mathrm{N})(1)$.

Circonférence moyenne $(\mathrm{Cg})$ et le diamètre moyen (Dg) - Correspond aux mesures de l'arbre de surface terrière moyenne :

$$
\mathrm{Cg}=\sqrt{\frac{\Sigma C^{2}}{N}} \quad \text { et } \quad \mathrm{Dg}=\frac{C g}{\pi}
$$

Avec C : somme des circonférences au carré de $\mathrm{N}$ arbres/ha.

Surface terrière (Gi) - C'est la somme des sections à $1,30 \mathrm{~m}$ du sol de l'ensemble des troncs des arbres qui composent 1 ha de peuplement. Cette grandeur est liée au volume sur pied du peuplement. Par exemple, pour un taillis, le produit de la surface terrière par sa hauteur donne une estimation de son volume en $\mathrm{m} 3$. Elle est exprimée en $\mathrm{m}^{2} /$ ha et est déterminée par la formule selon Favrichon et al. (1998) (1).

$$
\mathbf{G i}=\sum_{i=1}^{N} \frac{C^{2}}{4 \pi}
$$

La surface terrière individuelle est notée $\mathrm{g}$. Si l'on considère les arbres comme cylindriques et si l'on note $\mathrm{C}$ leur circonférence à $1,30 \mathrm{~m}$, (manuel Inventaire de l'ONF, Duplat et Perrotte 1981). (22) on a :

$$
\mathrm{g}=\frac{C^{2}}{4 \pi}
$$

$\checkmark \quad$ Richesse floristique - La richesse floristique est définie comme le nombre d'espèces recensées à l'intérieur des limites d'un territoire, compte tenu de sa surface. Il est l'un des importants indicateurs de la diversité biologique. 


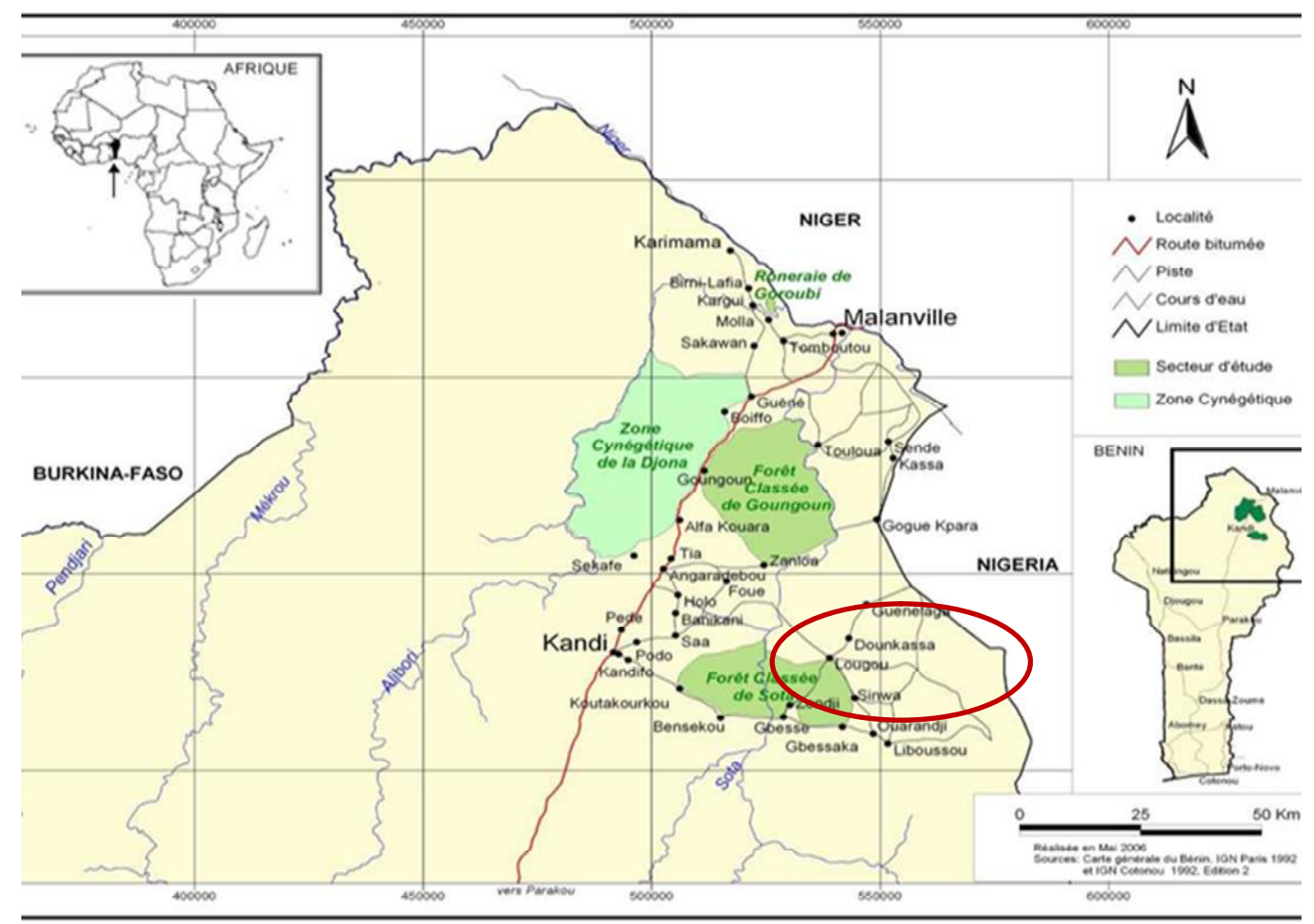

Figure 1: Carte de localisation de la forêt classée de la Sota.

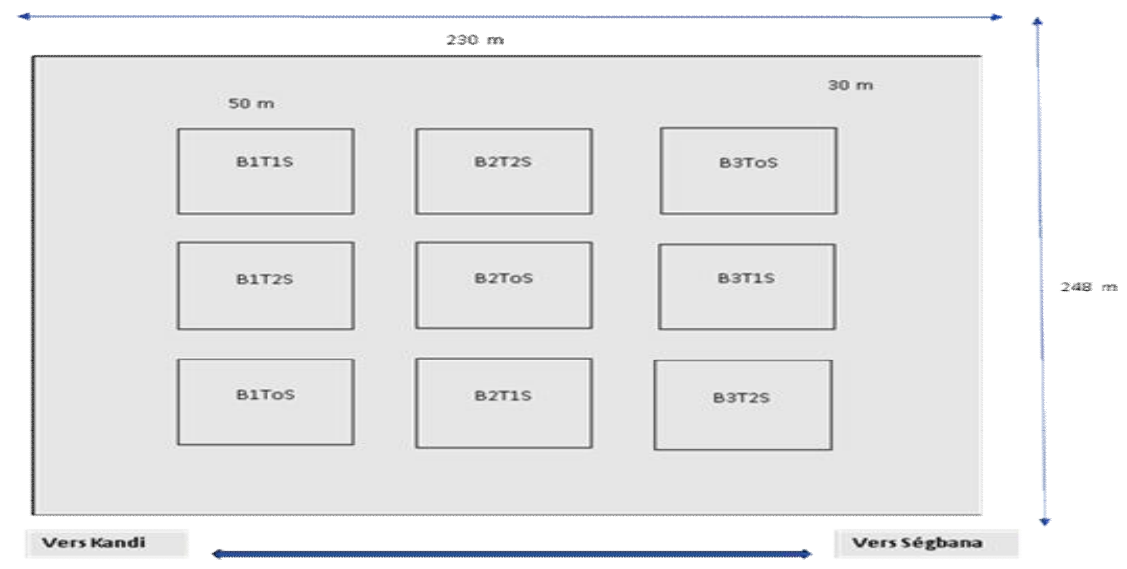

Figure 2 : Schéma du dispositif expérimental.

\section{RÉSULTATS}

Richesse floristique

La Figure 3 montre la composition floristique et l'abondance (en pourcentage) des essences inventoriée sur les Parcelles d'essais Sylvicoles de Bensékou. L'analyse de la Figure 3 a montré que les PES de Bensékou se retrouve dans une savane arbustive composé majoritairement des espèces comme Detarium microcarpum, Terminalia avicennioides, Vittelaria paradoxa, Combretum racemosum, Burkea afircana et en 
minorité de Prosopis africana, Daniella oliverie, Parkia biglobosa, Lannea spp, Pterocarpus erinaceus et Vitex doniana. Ces espèces représentent les caractéristiques d'une savane arbustive comprenant pas des ligneux de plus de cinq (5) mètres de haut et qui sont constitués essentiellement d'arbustes ne de buissons et beaucoup d'herbes spécifiquement des graminées (Figure 4).

\section{Densité du peuplement sur le site des PES Évolution générale de la densité moyenne du peuplement}

La Figure 5 montre l'évolution de la densité moyenne du peuplement sur le site des PES suivant les années. Elle pourrait révéler que la densité moyenne a augmenté en général d'environ 604 pieds/ha entre 1996 et 2014. Mais de façon spécifique, cette augmentation étant plus accentuée voire rapide dans la période 1996-2001 (200 pieds/ha de plus) que dans la période 2001-2014 (111 pieds/ha). L'augmentation de la densité moyenne observée entre 1996-2014 pourrait s'expliquer par la régénération naturelle et les rejets de souches des essences. En effet, la régénération naturelle a entrainé la multiplication de plusieurs jeunes arbres à faible diamètre dont le nombre important par unité de surface a fait augmenter la densité. De plus, après l'application des traitements en taillis simple, plusieurs rejets peuvent émergés d'une seule souche participant à l'augmentation substantielle de la densité.

\section{Evolution de la densité moyenne suivant les traitements}

La Figure 6 qui montre l'évolution de la densité du peuplement par traitement en fonction des années sur le site des PES de Bensékou. a révélé qu'au niveau des traitements T1 et T2 (T3), la densité moyenne a connu une augmentation soutenue d'environ 772 pieds/ha entre 1996 et 2014. De 19962009 la densité moyenne a été des 688 pieds/ha au sein de ces parcelles et entre 2009 et 2014, elle a connu une baisse considérable de 84 pieds/ha. Par contre, dans le cas du témoin To, on a remarqué que la densité moyenne a connu une augmentation de 768 pieds/ha entre 1996-2009. Mais entre 20092014, une baisse brute a été observée d'environ 339 pieds/ha. De 1996 et 2014 la densité moyenne a été de 428 pieds/ha, ce qui est inférieur à celle du traitement T3 (772 pieds/ha). Les résultats obtenus à l'issus du traitement T3 pourraient s'expliquer par la forte capacité de régénération des formations car, en dépit de la forte pression anthropique, la densité du peuplement passe d'environ 352 pieds/ha à près de 1124 pieds/ha soit trois fois la densité initiales en près de 18 ans malgré les coupes anarchiques et la forte exploitation.

\section{Évolution de la surface terrière du peuplement sur le site des PES}

L'accroissement de la surface terrière moyenne observée entre 1996-2001 (Figure 7) s'expliquer par la croissance en diamètre des arbres et par la régénération naturelle et les rejets de souches des essences. En effet, durant cette période, le projet UNSO était encore opérationnel jusqu'à son terme en 1997. Les stratégies et dispositifs qui ont été mise en place pour la surveillance et le contrôle des PES se sont encore montré efficace contre la pression anthropique même après la fin dudit projet jusqu'en 2004.

L'analyse des données a montré clairement que la surface terrière moyenne a augmenté d'environ $20 \mathrm{~m}^{2} / \mathrm{ha}$ (de 3,21 à $23,21 \mathrm{~m}^{2} / \mathrm{ha}$ ) entre 1996 et 2001. Mais au-delà, c'est-à-dire entre 2001 et 2014, elle chute brusquement pour avoisiner les $2.68 \mathrm{~m}^{2} / \mathrm{ha}$ en 2014 (Figure 7).

Par contre, au cours de la période de 2009-2014, la chute cruelle de la surface terrière pourrait être due aux effets conjugués des feux tardifs à la fin du projet et la pression anthropique. Mais, la régénération naturelle a entraîné la multiplication de plusieurs jeunes arbres à faible diamètre dont le nombre important par unité de surface a fait augmenter la densité pendant les périodes de 1996 à 2001 qui est l'année d'inflexion. Toutefois, l'application des traitements en taillis simple sur plusieurs individus du peuplement pourrait faire émerger les souches participant à l'augmentation substantielle de la densité. 


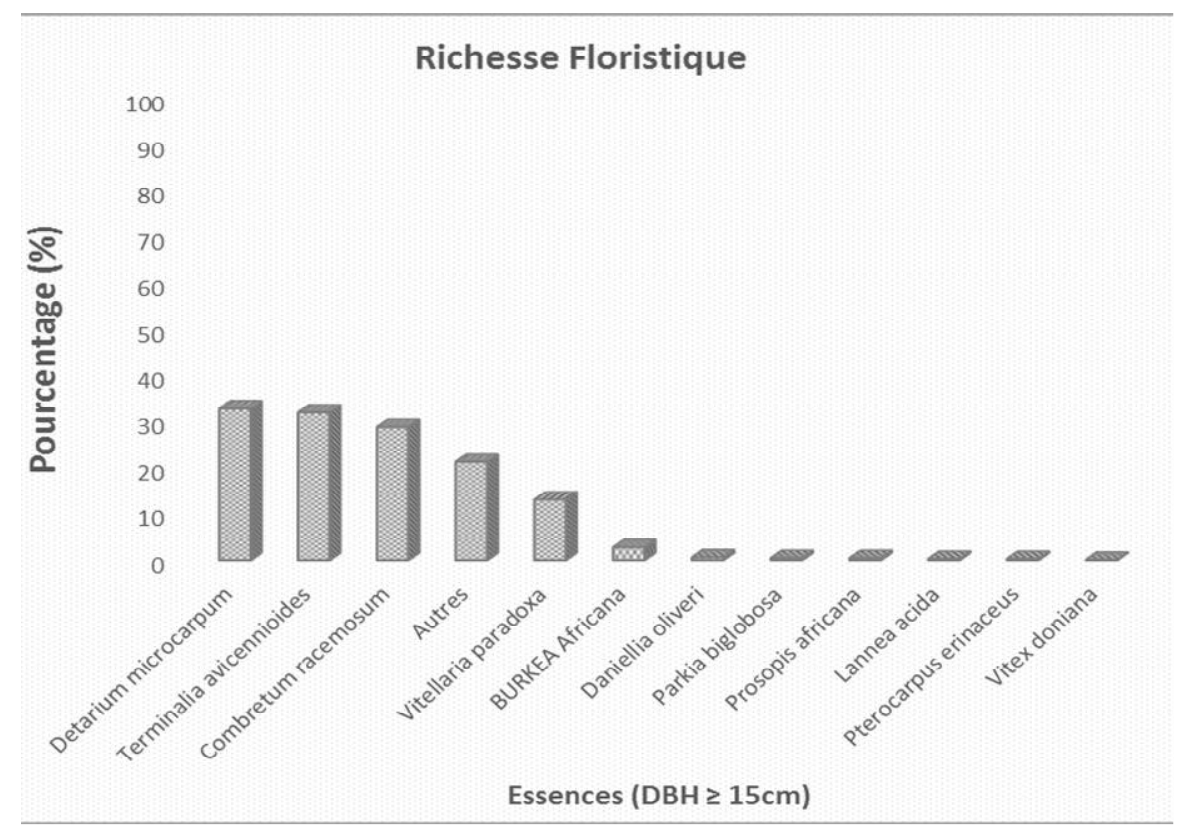

Figure 3 : Composition floristiques des Parcelles d'essais Sylvicoles de Bensékou.
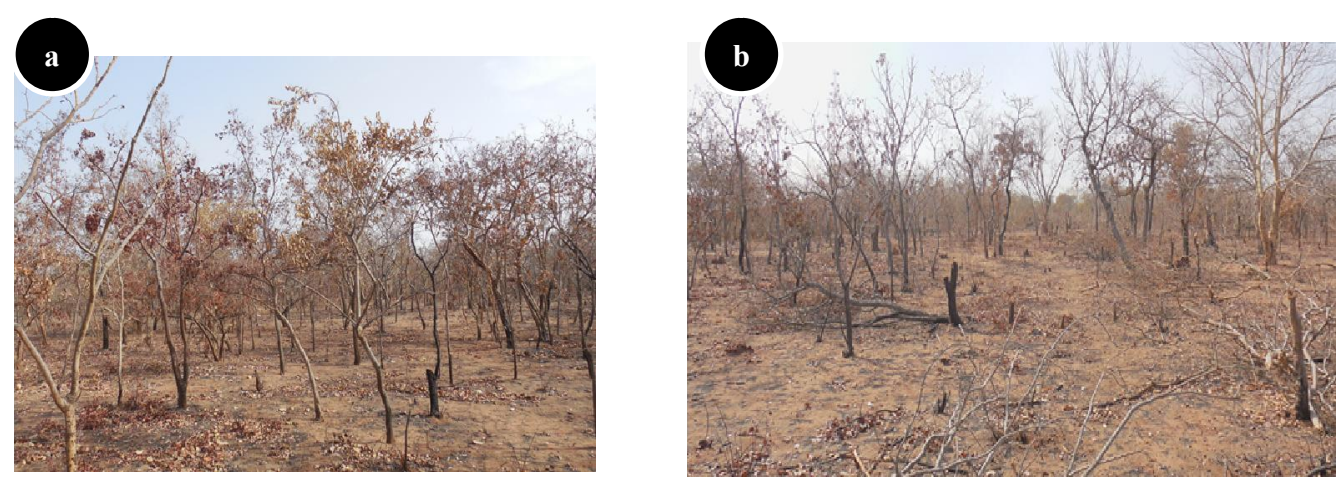

Figure 4: Photo a et b montrant la formation végétale sur les PES de Bensékou.

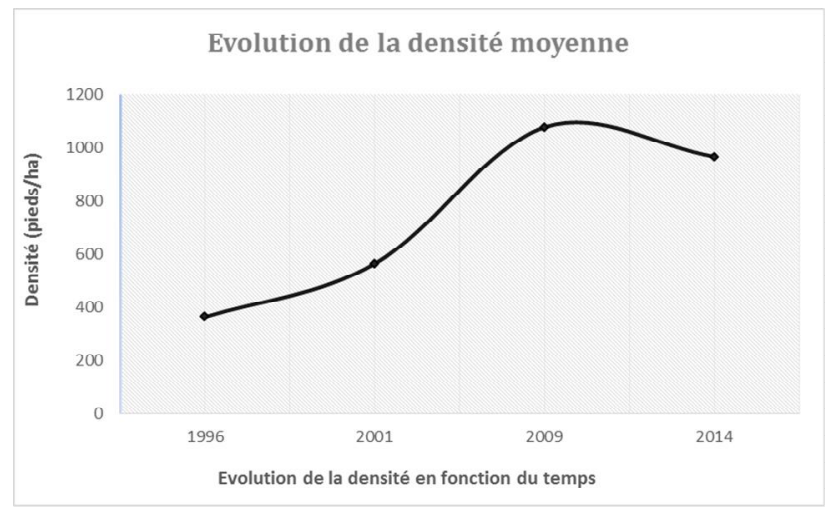

Figure 5 : Évolution de la densité moyenne du peuplement suivant les années. 


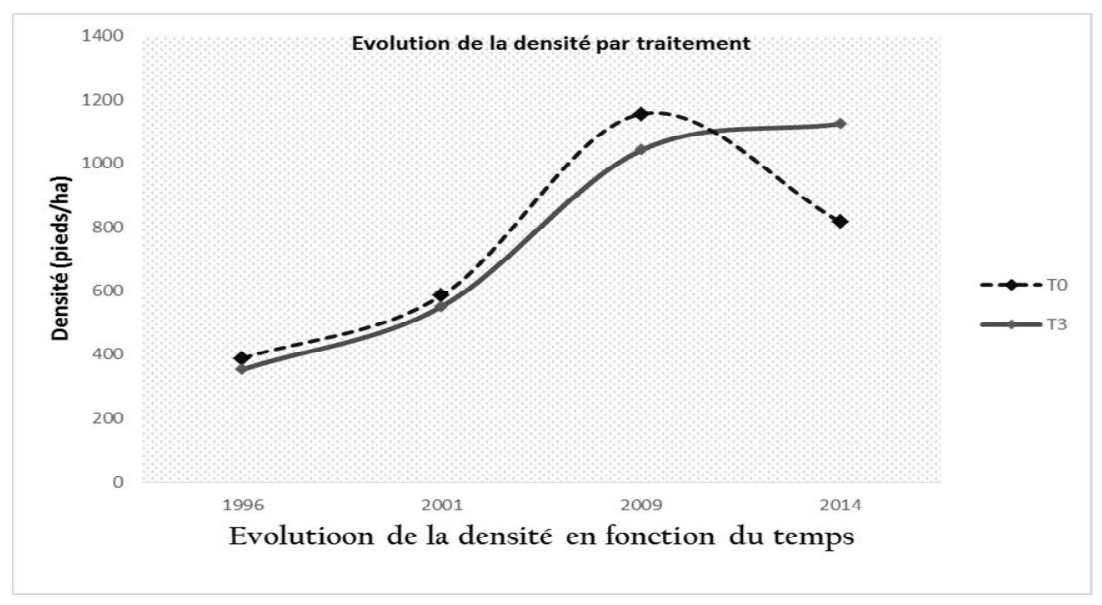

Figure 6 : Évolution de la densité moyenne sous les traitements (To et T1) suivant les années.

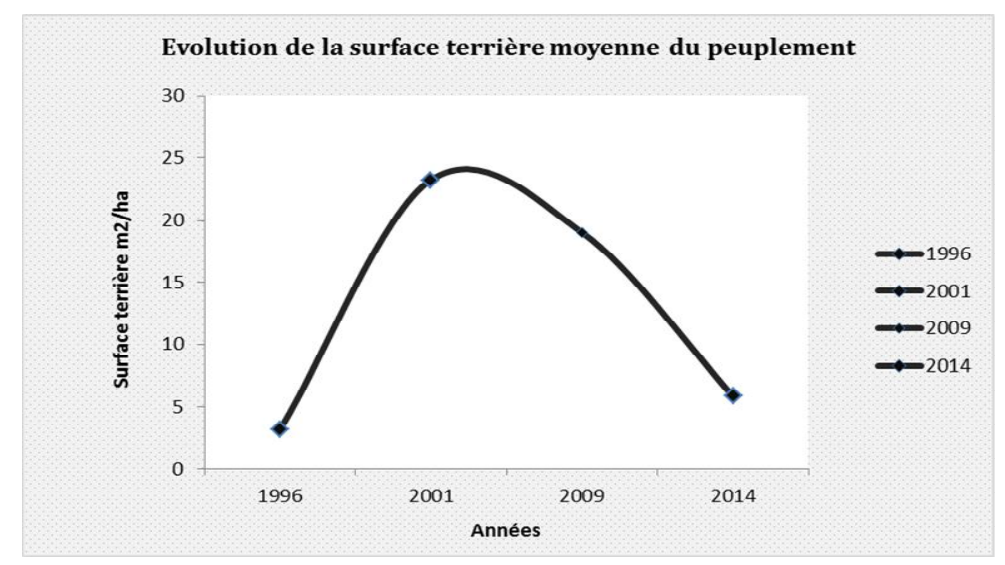

Figure 7 : Évolution de la surface terrière moyenne du peuplement suivant les années.

\section{DISCUSSION}

Le présent travail nous a permis d'évaluer la dynamique des Parcelles d'Essais Sylvicoles (PES) installées dans la Forêt Classée de la Sota en zone soudanienne, de faire l'étude de l'évolution du peuplement au sein des deux parcelles (T0 et $\mathrm{T} 3$ ); afin de dégager les principaux facteurs qui influencent leurs viabilités.

\section{Richesse floristique}

L'analyse floristique sur le site d'expérimentions des PES de Bensékou a permis de recenser les essences comme: Detarium microcarpum, Terminalia avicennioides, Vittelaria paradoxa,
Combretum racemosum, Prosopis africana, Daniella oliverie, Parkia biglobosa, Pterocarpus erinaceus et Vitex doniana. Ces différentes espèces sont caractéristiques d'une savane arbustive. La famille la plus représentée est celle des Fabaceae. Houinato (2001) obtient des résultats similaires sur le caractère représentatif de ces familles dans cette région soudanienne. Adjanohoun et al. (1967) et Guillaumet et al. (1971) avaient déjà eu des résultats similaires mais ont montré que la composition floristique de la savane étudiée à pour famille dominante les Fabaceae. Ce résultat est conforme à la description de toute la zone soudanienne. Koulibaly et al. (2006) abondent dans le même sens que les trois 
précédents auteurs. Les espèces fréquemment enregistrés sont: Detarium microcarpum, Terminalia spp., Combretum spp., Daniellia Oliveri, Parinari curatellifolia et Andropogon spp., dans la végétation de savane soudanienne (Adjanohoun et al., 1967 ; Cesar, 1978 ; White, 1986 ; Fournier, 1991 ; Mitja, 1992 ; Neumann et al., 1999; Reevineau, 2001). Selon Atindogbe (2004) la composition floristique des sites étudiés, caractérisée par la prédominance des espèces soudaniennes, confirme l'appartenance de ces formations à la zone soudanienne.

\section{Évolution générale de la densité moyenne du peuplement}

La densité moyenne du peuplement passe d'environ 352 pieds/ha à près de 1124 pieds/ha soit trois fois la densité initiale en près de 18 ans malgré les coupes anarchiques et la forte exploitation. Cette large augmentation pourrait s'expliquer par la forte capacité de régénération de cette forêt composée essentiellement de plantes à rejets.

La densité du peuplement par traitement en fonction des années a révélé qu'au niveau des traitements $T_{3}$ où les taillis simples et les feux de végétation ont été appliqués, la densité moyenne varie entre 688 et 772 de 1996-2009 pieds/ha pour tous les traitements. Ce qui correspond aux résultats relatifs à la moyenne de 745,2 individus/ha retrouvé par Maazou et al. (2017) au sein des mêmes types de formations dans la commune rurale de Dantchandou au Niger. Par ailleurs, la densité connaît une baisse considérable de 84 pieds/ha à la fin du projet (2009-2014) dans la parcelle $T_{3}$. Cette baisse est encore plus remarquable (environ 339 pieds/ha) sur les parcelles témoins $T_{0}$. Ces résultats peuvent être expliqués par l'abandon et le non suivi du peuplement pendant plusieurs années après la fin du projet.

\section{Évolution de la surface terrière du peuplement sur le site des PES}

Entre 1996-2001 la croissance en diamètre des arbres a été rapide grâce à la régénération naturelle et aux rejets de souches des essences, ce qui a permis l'accroissement de la surface terrière moyenne sur les PES durant la période d'exécution du projet UNSO. Par contre, entre 2001-2014, une chute cruelle de la surface terrière a été observée et est certainement due aux effets conjugués des feux tardifs et du non suivi des PES à la fin du projet. Mais, la régénération naturelle et l'émergence des souches a entraîné la multiplication de plusieurs jeunes arbres à faible diamètre dans les traitements $\mathrm{T}_{0}$ et $\mathrm{T}_{3}$. Nouvellet (1993) remarque les mêmes résultats en observant le pic au niveau de la classe 10 à $20 \mathrm{~cm}$ de circonférence caractéristique du peuplement jeune constitué essentiellement de rejet avec une surface terrière qui donne $2,95 \mathrm{~m}^{2} / \mathrm{ha} /$ an sur le même site. L'observation de la surface terrière dans le futur sera particulièrement intéressante pour suivre cette évolution. Les études menées dans la forêt de Gonré donnaient des résultats semblables pour un peuplement de quatre ans (Bonkoungou et al., 1988). La surface terrière des essences du peuplement exploité ou éclairci évolue favorablement par rapport au peuplement témoin au cours des quatre premières années qui suivent le traitement sylvicole (éclaircie ou exploitation). Le passage du feu inverse la situation : on observe une destruction de la surface terrière dans toutes les parcelles (Agonyissa et al., 1998).

\section{Conclusion}

L'inventaire réalisé sur les PES de la Sota combiné aux données historiques a permis de connaître la dynamique de ses peuplements. L'étude floristique a permis de rassembler, de synthétiser et de présenter les résultats de la recherche sur la dynamique des peuplements dans la forêt classée de Bensékou avec pour intérêt la caractérisation et l'estimation de l'utilisation future des parcelles des peuplements forestiers de la forêt classée de la Sota. Ainsi, au cours des cinq premières années (1996-2001), la densité et la surface moyenne du peuplement ont connu une augmentation. Mais à la fin du projet et avec le manque d'entretien et de suivi, ces paramètres ont chuté considérablement mise à part la densité dont 
la diminution est constatée plus tard après 2009. Néanmoins, elle présente un intérêt économique, ce qui s'explique par la régénération des jeunes pousses. En somme, dix-huit ans après l'application des traitements sylvicoles expérimentaux, le bilan floristique de la forêt classée de la Sota se traduit par une excellente reconstitution du couvert forestier supérieur mais la pression anthropique constitue un handicap pour sa stabilité.

\section{CONFLIT D'INTERETS}

Les auteurs déclarent qu'ils n'ont aucun conflit d'intérêts concernant cet article.

\section{CONTRIBUTIONS DES AUTEURS}

GSA est l'investigateur principal; RSL a contribué dans l'analyse des données ; ISL a participé à la collecte et à la rédaction de l'article; ICB et LZY ont participé à la collecte des données.

\section{ACKNOWLEDGMENTS}

Nous remercions le projet UNSO aménagement participatif des forets pour la séquestration du carbone et la Direction des eaux et Forêts pour avoir facilité la mise en œuvre ledit Projet.

\section{REFERENCES}

Adjanohoun E, Aké Assi L. 1967. Inventaire floristique des forêts claires soussoudanaises en Côte-d'Ivoire septentrionale. Annale Faculté Science Abidjan, 3: 85-148.

Agonyissa D, Sinsin B. 1998. Productivité et capacité de charge des pâturages au Bénin. Revue Elevage et Médecine Vétérinaire des pays tropicaux, 51(3) : 239-246.

Akouehou GS, Houndonougbo A, Tente B. 2013. La dynamique des systèmes de production dans les terroirs agricoles riverains de la forêt intercommunale de Fita-Agbado dans les communes de Dassa-Zoumé et de Savalou, Département des Collines au Centre du Bénin. International Journal of Biological and Chemical Sciences, 7(5):
1877-1891. DOI : http://dx.doi.org/ 10.4314/ijbcs.v7i5.8

Alexandre DY. 1989. Dynamique de la Régénération Naturelle en Forêt Dense de la Côte d'Ivoire. ORSTOM : Paris; 102.

Atindogbe G. 2004. Analyse de la structure spatiale des arbres: Application à l'enrichissement des savanes boisées de la zone soudanienne au Bénin. Thèse d'ingénieur Agronome, Université d'Abomey-Calavi, Bénin, 97p.

Tiokeng B, Mapongmetsem P-M, Nguetsop VF, Tacham WN. 2015. Biodiversité floristique et régénération naturelle sur les Hautes Terre de Lebialem (Ouest Cameroun). International Journal of Biological and Chemical Sciences, 9(1): 56-68. DOI: http://dx.doi.org/10.4314/ijbcs.v9i1.6

Bonkoungou ED, De Framond H. 1988. Dynamique du peuplement et évolution de la productivité d'une parcelle de formation naturelle en forêt de Gonsé. B.F.T, 218: 63-70.

Cesar J. 1978. Végétation, flore et valeur pastorale des savanes du Parc National de la Comoé. Centre de Recherches Zootechniques de Minankro-Bouaké. Ministère de la Recherche Scientifique en Côte d'Ivoire, 28p.

Yehouenou DRT, Akouehou GS, Ganglo JC. 2012. Caractéristiques structurales et écologiques des populations de Antiaris toxicaria (Pers.) Lesch et de Ceiba pentandra (L.) Gaertn dans les forêts reliques du Sud-Benin. International Journal of Biological and Chemical Sciences, 6(6): 5056-5067. DOI: http://dx.doi.org/10.4314/ijbcs.v6i6.24

Duplat P, Perrotte G. 1981. Inventaire et Estimation de l'Accroissement des Peuplements Forestiers. Office National des Forêts : Paris.

Fandohan AB. 2007. Structure des populations et importance socioculturelle du tamarinier (Tamatindus indica L.) dans la commune de Karimama (Bénin), Faculté des Sciences Agronomiques., 
Université Abomey-Calavi, Bénin, Mémoire de DEA, 55 p.

Favrichon V, Gourlet-Fleury S, Bar-Hen A, Dessard H. 1998. Parcelles Permanentes de Recherche en Forêt Dense Tropicale Humide (Eléments pour une Méthodologie d'Analyse des Données). CIRAD-Forêt, Campus International de Baillarguet : Montpellier, France ; 73.

Fournier A. 1991. Phénologie, croissance et production Végétales Dans Quelques savanes d'Afrique de l'Ouest. Variation selon un gradient climatique. Thèse de doctorat. Université de Paris VI, 246p.

Frontier S, Pichod-Viale D. 1993. Ecosystèmes: Structure, Fonctionnement, Evolution ( $2^{\mathrm{e}}$ édn). Collection d'Ecologie 21. Masson: Paris ; 447.

Guillaumet J-L, Adjanohoun E. 1971. La végétation [de la Côte d'Ivoire]. Mémoire Orstom, 50: 157-263.

Houinato M. 2001. Phytosociologie, écologie, production et capacité de charge des formations végétales pâturées dans la région des Monts Kouffé (Bénin). Thèse Doctorat. Faculté Science. Laboratoire Botanique Systématique et phytosociologie. Université Libre Bruxelles, Belgique. 219p.

Koulibaly A, Goetze D, Traoré D, Porembsk S. 2006. Caractéristiques de la soudanienne: protégées contre savanes exploitées la végétation en Côte-d'Ivoire. Candollea, 61(2): 425-452.

Mitja D. 1992. Influence de la culture itinérante sur la végétation d'une savane humide de Côte-d'Ivoire (Booro-Borotou - Touba). Paris : ORSTOM, (Etudes et Thèses). Th. : Biol. Végétale Tropicale, Université de Paris 6 : Paris.

N'Gueguim JR, Zapfack L, Youmbi E, Riera B, Onana J, Foahom B, Makombu GJ.
2009. Diversité floristique sous canopée en plantation forestière de MangombeEdea (Cameroun). Article: Biotechnologie, Agronomie, Société et Environnement, 14(1): 167-176.

Neumann K, Meuller-Haude P. 1999. Au le sud-ouest de Forêts du Burkina Faso: végétation - sols - l'action de l'homme. Phytocoenologia, 29: 53-85.

Nouvellet Y. 1993. Evolution d'un taillis de formation naturelle soudano-sahélienne au Burkina Faso. Résultats préliminaires à cinq ans Bois et Forêt des Tropiques, $\mathrm{n}^{\circ} 237,3 \mathrm{e}$ trimestre, $15 \mathrm{p}$.

Rahila Maazou, Habou Rabiou, Youssoufa Issiaka, Lawali Abdou, Sani Idi Saidou Ali Mahamane 2017. Influence de l'occupation des terres sur la dynamique des communautés végétales en zone Sahélienne : cas de la commune rurale de Dantchandou (Niger). International Journal of Biological and Chemical Sciences, 11(1): 79-92. DOI : http://dx.doi.org/10.4314/ijbcs.v11i1.7

Reevineau J-L. 2001. Les Espèces ligneuses indicatrices des sols Dans les savanes et jachères de l'Ouest du Burkina Faso. Phytocoenologia, 31: 325-351.

Tokpon C. 2010. Contribution à l'étude de la productivité des formations naturelles sèches (savanicoles) en vue de la production du bois énergie: Cas de la forêt classée de Sota. Mémoire de licence, $32 \mathrm{p}$.

White F. 1986. La végétation de l'Afrique. Adaptation française $P$. Bamps, ORSTOM et UNESCO, Paris. Coll. Recherches sur les ressources naturelles, $384 \mathrm{p}$.

Zobel BJ, Van Buijtenen JP. 1989. Wood Variation - Its Causes and Control. Springer-Verlag : Berlin. 